\title{
Synthesis, Docking Studies, Pharmacological Activity and Toxicity of a Novel Pyrazole Derivative (LQFM 021)—Possible Effects on Phosphodiesterase
}

\author{
Daniella Ramos Martins, ${ }^{a}$ Francine Pazini, ${ }^{b}$ Vinícius de Medeiros Alves, ${ }^{a}$ \\ Soraya Santana de Moura, ${ }^{a}$ Luciano Morais Lião, ${ }^{b}$ Mariana Torquato Quezado de Magalhães, ${ }^{c}$ \\ Marize Campos Valadares, ${ }^{a}$ Carolina Horta Andrade, ${ }^{a}$ Ricardo Menegatti, ${ }^{a}$ and \\ Matheus Lavorenti Rocha*,a \\ ${ }^{a}$ Faculty of Pharmacy, Federal University of Goias; Av. Universitária s/n, 74605-220 Goiânia, GO, Brazil: \\ ${ }^{b}$ Chemistry Institute, Federal University of Goias-Campus Samambaia; CP 131-74001-970 Goiânia, GO, \\ Brazil: and ${ }^{c}$ Laboratory of Mass Spectrometry (LEM), Embrapa Recursos Genéticos e Biotecnologia Brasília; \\ 70849-970-Brasilia, DF-Brazil. Received November 23, 2012; accepted February 5, 2013
}

This study describes the synthetic route and molecular computational docking of LQFM 021, as well as examines its biological effects and toxicity. The docking studies revealed strong interaction of LQFM 021 to phosphodiesterase-3 (PDE-3). In isolated arteries, the presence of endothelium potentiates the relaxation for LQFM 021 and the inhibition cyclic nucleotides reduced the relaxation. Pre-contraction with $\mathrm{KCl}(45 \mathrm{~mm})$, the treatment with tetraethylammonium (TEA) $(5 \mathrm{~mm})$ and inhibition of reticular $\mathrm{Ca}^{2+}$-ATPase showed an inhibitory effect on relaxation. Moreover, the compound reduced the contraction evoked by the $\mathrm{Ca}^{2+}$ influx. Acute toxicity tests revealed that the compound was practically nontoxic. In conclusion, this study showed that a new synthetic derivative of pyrazole is a possible PDE-3 inhibitor and has vasorelaxant activity and low toxicity.

Key words pyrazole; phosphodiesterase; relaxation; aorta; cyclic nucleotide

Pyrazoles are chemical compounds of synthetic origin that have a five-membered heterocycle with two nitrogen atoms and three adjacent carbons. Several members of the pyrazoles class have shown good pharmacological effects, as well as anti-inflammatory, analgesic, antipyretic, antiarrhythmic and tranquilizer activities. ${ }^{1,2}$ The activity of these compounds on vascular smooth muscle has not yet been well explored.

Increase in the intracellular concentrations of $3^{\prime}, 5^{\prime}$-cyclic adenosine monophosphate (cAMP) and $3^{\prime}, 5^{\prime}$-cyclic guanosine monophosphate (cGMP) lead to relaxation of vascular smooth muscle by reducing the concentration of intracellular $\mathrm{Ca}^{2+3}$. Degradation by cyclic nucleotide hydrolytic cleavage is catalyzed by phosphodiesterases (PDEs), ${ }^{4,5}$ which are divided into at least 11 different subtypes, PDE-1 to PDE-11, depending on the nucleotide that is hydrolyzed and the regulatory properties of the enzyme. ${ }^{6,7)}$ Some PDEs, including PDE-3, are responsible for inducing vascular relaxation in different vascular beds when they are inhibited, including in aortas of rats. ${ }^{8-10)}$

Inhibitors of PDEs increase cytosolic levels of cAMP and cGMP and cause vasodilation by activating protein kinases A (PKA) and G (PKG). This activation induces many cellular effects, such as the uptake of intracellular $\mathrm{Ca}^{2+}$ by the sarcoplasmic reticulum, dephosphorylation of the myosin light chain, inhibition of $\mathrm{Ca}^{2+}$ influx, activation of $\mathrm{K}^{+}$channels and hyperpolarization of the plasma membrane. ${ }^{10-12)}$ These processes lead to the relaxation of smooth muscle by decreasing the cytosolic concentration of $\mathrm{Ca}^{2+}$ and decreasing the sensitivity of the contractile apparatus to $\mathrm{Ca}^{2+} \cdot{ }^{2+}$ However, some inhibitors of PDEs can relax vascular smooth muscle through pathways that are independent of cyclic nucleotides. ${ }^{10,14,15)}$ Thus, it is important to investigate alternative pathways of relaxation that are dependent or independent of cyclic

The authors declare no conflict of interest nucleotides.

In the scope of a research program aimed at drug development for treatment of cardiovascular disease and stroke, we describe in the present study the synthesis and biological evaluation of new LQFM 021 heterocyclic derivative. LQFM 021 was originally designed through hybridization molecular from milrinone and cilostazol which are characterized as phosphodiesterase 3 inhibitors. The present work details the synthesis of LQFM 021 that is designed to be a PDE inhibitor, as well as its biological activity on vascular smooth muscle, molecular docking studies and toxicity analysis.

\section{Experimental}

Synthesis Steps. Synthesis of 1-(3-Fluorophenyl)-1H-pyrazole-4-carbonitrile (5) To a stirred heterogeneous mixture of $\mathrm{NH}_{2} \mathrm{OH} \cdot \mathrm{HCl}(1.3 \mathrm{mmol})$ and $\mathrm{NaI}(4 \mathrm{mmol})$ in $N, N$-dimethylformamide (DMF) (4 mL), 1-(3-fluorophenyl)-1 $H$-pyrazole4-carbaldehyde (4) $(1 \mathrm{mmol})$ was added at room temperature. The reaction mixture was heated at reflux temperature for $6 \mathrm{~h}$ and was then allowed to cool and poured into ice. The precipitate was vacuum filtered and dried, and the crude product was purified by chromatography using $\mathrm{CHCl}_{3}$ as mobile phase to provide 1-(3-fluorophenyl)-1H-pyrazole-4-carbonitrile (5) as a beige solid in $99 \%$ of yield: $\mathrm{mp} 115^{\circ} \mathrm{C}, R f=0.80$ ( $n$-hexane/ ethyl acetate, $7: 3): \mathrm{IR}_{\max }(\mathrm{KBr}) \mathrm{cm}^{-1}: 3154(v \mathrm{C}-\mathrm{H}), 2235$ $(v \mathrm{C}-\mathrm{N}), 864$ (v 1,3-C-F); ${ }^{1} \mathrm{H}-\mathrm{NMR}(500.13 \mathrm{MHz}) \mathrm{CDCl}_{3} \delta$ : $8.32(1 \mathrm{H}, \mathrm{d}, J=0.6 \mathrm{~Hz}, \mathrm{H}-5), 8.00(1 \mathrm{H}, \mathrm{d}, J=0.6 \mathrm{~Hz}, \mathrm{H}-3), 7.49$ $\left(1 \mathrm{H}\right.$, dddd, $J=8.1,2.3,1.4$ and $\left.0.5 \mathrm{~Hz}, \mathrm{H}-6^{\prime}\right), 7.47$ (1H, dddd, $J=10.3,2.4,2.3$ and $\left.0.6 \mathrm{~Hz}, \mathrm{H}-2^{\prime}\right), 7.46(1 \mathrm{H}$, dddd, $J=8.1$, $7.8,5.4$ and $\left.0.6 \mathrm{~Hz}, \mathrm{H}-5^{\prime}\right), 7.12(1 \mathrm{H}$, dddd, $J=8.0,7.8,2.4$ and $\left.1.4 \mathrm{~Hz}, \mathrm{H}-4^{\prime}\right) ;{ }^{13} \mathrm{C}-\mathrm{NMR}(125.76 \mathrm{MHz}) \mathrm{CDCl}_{3} \delta$ : $163.2\left(\mathrm{C}-3^{\prime}\right)$, 143.5 (C-3), $139.9\left(\mathrm{C}-1^{\prime}\right), 131.9$ (C-5), $131.3\left(\mathrm{C}-5^{\prime}\right), 115.3\left(\mathrm{C}-4^{\prime}\right)$, $115.1\left(\mathrm{C}-6^{\prime}\right), 112.6(\mathrm{CN}), 107.9\left(\mathrm{C}-2^{\prime}\right), 94.9(\mathrm{C}-4) .[\mathrm{M}+\mathrm{H}]^{+} m / z$ $=188.058$. 
Synthesis of 5-(1-(3-Fluorophenyl)-1H-pyrazol-4-yl)-2Htetrazole (6) A mixture of 1-(3-fluorophenyl)-1 $H$-pyrazole4-carbonitrile (5) $(2.0 \mathrm{~g}, 12.4 \mathrm{mmol})$, sodium azide ( $4.1 \mathrm{~g}$, $62 \mathrm{mmol})$, and ammonium chloride $(3.35 \mathrm{~g}, 62 \mathrm{mmol})$ in $35 \mathrm{~mL}$ of DMF was heated at reflux temperature for $72 \mathrm{~h}$. The reaction mixture was then poured into water and acidified to $\mathrm{pH}$ 5. The product was vacuum filtered and dried to provide 5-(1-(3-fluorophenyl)- $1 H$-pyrazol-4-yl)-2 $H$-tetrazole (6) as a beige solid in $99 \%$ of yield; $\mathrm{mp} 205^{\circ} \mathrm{C}, \mathrm{Rf}=0.10\left(\mathrm{CH}_{2} \mathrm{Cl}_{2} /\right.$ $\mathrm{MeOH}, 95: 5): \mathrm{IR}_{\max }(\mathrm{KBr}) \mathrm{cm}^{-1}: 3399(v \mathrm{~N}-\mathrm{H}), 3124(v \mathrm{C}-\mathrm{H})$, $1631,1603(v \mathrm{C}=\mathrm{C}), 861(v 1,3-\mathrm{C}-\mathrm{F}) ;{ }^{1} \mathrm{H}-\mathrm{NMR}(500.13 \mathrm{MHz})$ DMSO- $d_{6} \delta: 9.25(1 \mathrm{H}, \mathrm{d}, J=0.7 \mathrm{~Hz}, \mathrm{H}-5), 8.34(1 \mathrm{H}, \mathrm{d}$, $J=0.7 \mathrm{~Hz}, \mathrm{H}-3), 7.85$ (1H, dddd, $J=8.0,2.1,0.9$ and $0.5 \mathrm{~Hz}$, $\left.\mathrm{H}-6^{\prime}\right), 7.84\left(1 \mathrm{H}\right.$, dddd, $J=10.0,2.5,2.1$ and $\left.0.9 \mathrm{~Hz}, \mathrm{H}-2^{\prime}\right), 7.61$ ( $1 \mathrm{H}$, dddd, $J=8.3,8.0,6.5$ and $\left.0.9 \mathrm{~Hz}, \mathrm{H}-5^{\prime}\right), 7.25$ (1H, dddd, $J=8.6,8.3,2.5$ and $\left.0.9 \mathrm{~Hz}, \mathrm{H}-4^{\prime}\right), 3.52$ (s, NH); ${ }^{13} \mathrm{C}-\mathrm{NMR}$ (125.76 MHz) DMSO- $d_{6} \delta$ : 162.6 (C-3'), 149.3 (C-1"), 140.3 $\left(\mathrm{C}-1^{\prime}\right), 139.7$ (C-3), $131.5\left(\mathrm{C}-5^{\prime}\right), 128.0(\mathrm{C}-5), 114.6\left(\mathrm{C}-2^{\prime}\right), 113.8$ $\left(\mathrm{C}-4^{\prime}\right), 108.9(\mathrm{C}-4), 106.2\left(\mathrm{C}-2^{\prime}\right) ;[\mathrm{M}+\mathrm{H}]^{+} m / z=231.060$.

Docking Studies The 2D structure of LQFM 021 was drawn using MarvinSketch v.5.5 (ChemAxon, Budapest, Hungary, www.chemaxon.com). Then, the OMEGA software ${ }^{16,17)}$ was used to generate $3 \mathrm{D}$ structures and torsion driving. All parameters used were the OMEGA default values.

The crystal structures of human phosphodiesterases PDE-3, PDE-4 and PDE-5 were retrieved from the Protein Data Bank (PDB). The accession code for PDE-3 in complex with MERCK1 with $2.40 \AA$ resolution is $1 \mathrm{SO} 2,{ }^{18)}$ the accession code for PDE-4 co-crystallized with rolipram with $2.00 \AA$ resolution is $1 \mathrm{RO} 6,{ }^{19)}$ and the accession code for PDE-5 co-crystallized with tadalafil with $1.37 \AA$ resolution is $1 \mathrm{XOZ}^{20)}$

For docking studies, the enzyme structures were prepared by first removing the ligand molecule from the active site and then by removing the water molecules, with the exception of the active site water molecules (wat11-wat16 for PDE-3; wat629, wat637, wat664, wat685, wat778 and wat788 for PDE-4; and wat1017, wat1128, wat1148, wat1169 and wat1232 for PDE-5), as indicated by the literature. ${ }^{18-20)}$ Then, AutoDockTools 4.0 version 1.5.2 was used to add hydrogen atoms to the enzyme structures. Partial atomic charges were then assigned to the macromolecule and ligands. Gasteiger charges were used for the ligands, and Kollman charges were used for the protein.

Docking calculations were performed in AutoDock 4.2 21 ) with the Genetic Algorithm and Local Search Parameters (GALS). The regions of interest in the enzyme were defined using AutoGrid software. A grid box centering in the cocrystallized ligands for each protein were generated using the following grid parameters: resolution of $40 \AA \times 50 \AA \times 40 \AA$ points, with a grid space of 0.375 and coordinates $58.4,2.4$, and 10.0 for the $x$-, $y$ - and $z$-axes for PDE-3; resolution of $40 \AA \times 40 \AA \times 50 \AA$ points, with a grid space of 0.375 and coordinates $32.3,75.9$, and 20.6 for the $x$-, $y$ - and $z$-axes for PDE-4; and $40 \AA \times 40 \AA \times 40 \AA$ points, with a grid space of 0.375 and coordinates 47.3, 35.1, and 13.0 for the $x$-, $y$ - and $z$-axes for PDE-5. All the rotatable single bonds in the ligands were allowed to rotate and we kept the protein structures rigid in the docking calculation. For analysis purpose, we clustered the generated docking solutions with the root-mean-square deviation (RMSD) cutoff set to $0.5 \AA$. Autodock generated 150 conformers of LQFM 021 docked with each protein, corresponding to the lowest-energy complex structures.

Study of Vascular Reactivity. Preparation of Aortic Rings Male Wistar rats (200-230g) from the central animal facility of the Federal University of Goiás were used in this study. All experiments were carried out in accordance with the Animal Research Ethical Committee of the Federal University of Goiás, Goiânia, GO, Brazil. The rats were anaesthetized and killed by abdominal aortic exsanguinations. The thoracic aorta was isolated and connective tissue and fat were removed. The isolated aortas were cut into rings approximately $4 \mathrm{~mm}$ long, placed between two stainless-steel stirrups and connected to an isometric force transducer. The responses were recorded using a computerized system and a WinDaq Resource (DATAQ Instruments, Akron, OH, U.S.A.) data acquisition unit to measure tension in the preparations. The aortic rings were placed in a $10 \mathrm{~mL}$ organ chamber containing Krebs solution with the following composition: $130 \mathrm{~mm}$ $\mathrm{NaCl}, 4.7 \mathrm{~mm} \mathrm{KCl}, 1.2 \mathrm{~mm} \mathrm{KH} \mathrm{KO}_{4}, 1.2 \mathrm{~mm} \mathrm{MgSO}_{4}, 14.9 \mathrm{~mm}$ $\mathrm{NaHCO}_{3}, 5.5 \mathrm{~mm}$ glucose, and $1.6 \mathrm{~mm} \mathrm{CaCl}$. The solution was maintained at $\mathrm{pH} 7.4$, and gassed with $95 \% \mathrm{O}_{2}$ and $5 \% \mathrm{CO}_{2}$ at $37^{\circ} \mathrm{C}$. The rings were initially stretched to a basal tension of $1.0 \mathrm{~g}$ (optimal basal tone, previously determined by lengthtension relationship experiments) before allowing them to equilibrate in the bathing medium. In some preparations, the endothelium was mechanically removed.

Experimental Procedure After $40 \mathrm{~min}$ of equilibration, cumulative concentration-response curves for LQFM $021(1 \mu \mathrm{M}$ to $300 \mu \mathrm{M})$ were carried out in isolated aortic rings with $\left(\mathrm{E}^{+}\right)$and without $\left(\mathrm{E}^{-}\right)$endothelium pre-contracted with phenylephrine $\left(0.1 \mu \mathrm{M}\right.$, the $\mathrm{EC}_{50}$ previously determined in our laboratory) or after contraction with $45 \mathrm{~mm} \mathrm{KCl}$. To investigate the mechanism(s) responsible for LQFM 021-induced relaxation, aortic rings were contracted with phenylephrine $(0.1 \mu \mathrm{M})$ $30 \mathrm{~min}$ after incubation with one of the following drugs: the selective soluble guanylyl cyclase inhibitor $1 H$-[1,2,4]oxadiazolo-[4,3-a]quinoxalin-1-one (ODQ, $1 \mu \mathrm{M})$, the selective adenylyl cyclase inhibitor $c i s-N$-(2-phenylcyclopentyl)-azacyclotridec-1-en-2-amine hydrochloride (MDL-12,330A, $10 \mu \mathrm{M}$ ), the non-selective $\mathrm{K}^{+}$channels blocker tetraethylammonium (TEA, $5 \mathrm{~mm}$ ), or the specific sarcoplasmic reticulum $\mathrm{Ca}^{2+}$ ATPase (SERCA) inhibitor cyclopiazonic acid (CPA, $10 \mu \mathrm{M})$.

To further analyze the relative contribution of LQFM 021 to the inhibition of the contraction evoked by $\mathrm{Ca}^{2+}$ influx, the role of extracellular $\mathrm{Ca}^{2+}$ mobilization was investigated in $\mathrm{CaCl}_{2}$-induced contraction in the presence of phenylephrine. Aortic rings were first contracted with $45 \mathrm{~mm} \mathrm{KCl}$ to obtain the maximal contraction in each preparation. After washing and returning to baseline, phenylephrine $(0.1 \mu \mathrm{M})$ was used to deplete the intracellular $\mathrm{Ca}^{2+}$ stores in $\mathrm{Ca}^{2+}$-free solution until the disappearance of any contractile response (approximately 4-5 stimuli with phenylephrine, each approximately $10 \mathrm{~min}$ ). The samples were then rinsed in $\mathrm{Ca}^{2+}$-free solution containing phenylephrine $(0.1 \mu \mathrm{M})$. Next, the cumulative concentrationresponse curves for $\mathrm{CaCl}_{2}(0.0-3.0 \mathrm{~mm})$ were obtained in the absence (control group) or after a $20 \mathrm{~min}$ incubation period with LQFM $021(100 \mu \mathrm{M})$. Each point is expressed as maximal change from the contraction produced by $\mathrm{KCl}(45 \mathrm{~mm})$, which was taken as $100 \%$.

Evaluation of Toxicity. Assay of Incorporation of Neutral Red in Basal Line 3T3 The viability of 3T3 cells after LQFM 021 treatment was evaluated using an assay of 


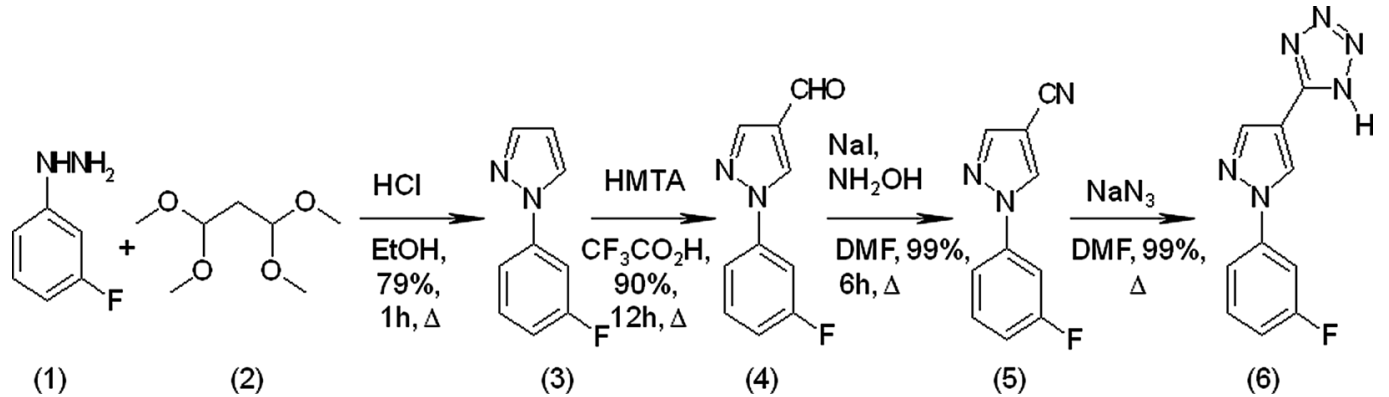

Fig. 1. Synthetic Route for the Preparation of 5-(1-(3-Fluorophenyl)-1H-pyrazol-4-yl)-2H-tetrazole (LQFM 021)

incorporation of neutral red dye. This dye is commonly used as an indicator of cytotoxicity and accumulates in the lysosomes of viable cells. ${ }^{22}$ This test can also be used to support dose selection in the evaluation of acute oral toxicity according to the Organization for Economic Cooperation and Development (OECD) 423 "Acute Oral Toxicity Class," 2001. For this purpose, the value of $\mathrm{IC}_{50}(\mu \mathrm{g} / \mathrm{mL})$ is used to estimate the $\mathrm{LD}_{50}(\mathrm{mg} / \mathrm{kg})$ according to the equation $\log \left(\mathrm{LD}_{50}\right)=0.435 \times \log$ $\left(\mathrm{IC}_{50}\right)+0.628$, validated by the Interagency Coordination Committee on the Validation of Alternative Methods (ICCVAM, 2006). For the test, the cells $\left(3 \times 10^{4}\right.$ cells $\left./ \mathrm{mL}\right)$ were seeded into 96-well flat microtiter plates (Corning, U.S.A.) in Dulbecco's modified Eagle's medium (DMEM) 1640 medium supplemented with $10 \%$ fetal bovine serum (FBS) and incubated for $24 \mathrm{~h}$. The cells were then incubated with or without eight concentrations $(0.005-0.602 \mathrm{mg} / \mathrm{mL})$ of LQFM 021 in sextuplicate for $48 \mathrm{~h}$ and were performed as previously described (ICCVAM, 2006). The absorbance was measured at $540 \mathrm{~nm}$ and the $\mathrm{IC}_{50}$ value obtained was utilized to support dose selection in the evaluation of acute oral toxicity.

Acute Oral Toxicity Evaluation of LQFM 021 The acute oral toxicity evaluation was performed according to the guide recommended in OECD 423 "Acute Oral Toxicity Class," 2001. The initial dose selected for LQFM 021 was $2000 \mathrm{mg}$ / $\mathrm{kg}$, set from data obtained in the in vitro test of the incorporation of neutral red with $3 \mathrm{~T} 3$ basal cells. LQFM 021 was prepared in edible vegetable oil (sunflower oil), and $0.2 \mathrm{~mL}$ was administered orally (gavage) to female Swiss mice. This dose was tested twice and thus estimated the category toxicology of the extract according to the specifications of the guide. The animals were fasted for $2 \mathrm{~h}$ before dosing. During the experimental period, the animals were observed continuously for $12 \mathrm{~h}$ and then observed daily for verification of any change in the general behavior or physiological activities. The animals were evaluated by the Hippocratic screening method, which determines the effects of LQFM 021 on the state of consciousness, disposal, activity and coordination of the motor system and muscle tone, as well as the activity of the autonomic and central nervous system of the animal. Namely, irritability, touch response, response to the tightening of tail, twisting, hindquarters position, righting reflex, power grab, headset reflex, corneal reflex, tremors, straub, anesthesia, lacrimation, ptosis eyelid, piloerection, cyanosis and death were all monitored. ${ }^{23,24)}$

Statistical Analysis In the graphics, the data are presented as the means \pm S.E.M. In each set of experiments, $n$ indicates the number of rats studied. The values for reactivity and response to LQFM 021 are expressed as percentage of the preceding contraction. The maximum relaxant effect $\left(E_{\max }\right)$ was considered as the maximal amplitude response reached in concentration-effect curves for LQFM 021. Statistical analyses were performed using GraphPad Prism version 5.0 (GraphPad Software Inc., San Diego, CA, U.S.A.). Comparisons among groups were performed using one-way ANOVA (post-test: Newman-Keuls) and Student's $t$-test, and values of $p<0.05$ were considered to be significant.

\section{Results}

Synthesis of LQFM 021 As illustrated in Fig. 1, the synthetic route began with 1-(3-fluorophenyl)-1 $H$-pyrazole (3) and proceeded through the classical method described by Finar and Godfrey. ${ }^{25)}$ Chemoselective and regiospecific formylation of 1-(3-fluorophenyl)- $1 H$-pyrazole (3) to 1-(3-fluorophenyl)- $1 H$ pyrazole-4-carbaldehyde (4) was performed under Duff's conditions. ${ }^{26,27)}$ The synthesis of 1-(3-fluorophenyl)-1H-pyrazole-4-carbonitrile (5) was carried out through the formation of the oxime by reaction of 1-(3-fluorophenyl)-1H-pyrazole4-carbaldehyde (4) with hydroxylamine. This was followed by in situ dehydration in the presence of sodium iodide and DMF at reflux temperature for $6 \mathrm{~h}$ to afford (5) a $99.0 \%$ yield. ${ }^{28)}$ 5-(1-(3-Fluorophenyl)-1H-pyrazol-4-yl)-2H-tetrazole, or LQFM 021 (6), was synthetized through a 1,3-bipolar cycloaddition between 1-(3-fluorophenyl)-1 $H$-pyrazole-4-carbonitrile (5) and $\mathrm{NaN}_{3}$ using $\mathrm{NH}_{4} \mathrm{Cl}$ as the catalyst in DMF at reflux temperature for $72 \mathrm{~h}$ in $99 \%$ yield. $^{29)}$ LQFM 021 (6) was obtained in a $69.6 \%$ overall yield.

Docking Studies To determine the efficiency of the docking parameters and the size of the grid box, the docking protocol was evaluated, using the same parameters of the docking procedure described in item 2.2, but with the co-crystallized ligand of each protein. Each co-crystalized ligand was redocked into the corresponding binding site, and the accuracy of each prediction was assessed based on the RMSD between the coordinates of the heavy atoms of the ligand in the top docking orientation and those in the crystal structure. The PDE-3-MERCK1 inhibitor complex (PDB ID: 1SO2) was used for the validation run of PDE-3, where MERCK1 was docked into PDE-3. Docking using the AutoDock software with the rigid protein structure accurately predicted the crystallographic placement of MERCK1 in the crystal structure of human PDE-3 with a RMSD of $0.85 \AA$. The same validation method was performed for PDE-4 with the PDE4-Rolipram complex (PDB ID: 1RO6) and for PDE-5 with the PDE5-Tadalafil complex (PDB ID: 1XOZ). The re-docking of rolipram in the human PDE-4 crystal structure showed a RMSD of $0.49 \AA$, while the re-docking of tadalafil in the human PDE-5 crystal 
structure showed a RMSD of $0.54 \AA$. The docking protocol was considered validated because it presented RMSD values equal to or less than $2.0 \AA$, as reported in the literature. ${ }^{30)}$ The

Table 1. Docking Results for the Best Conformations of LQFM 021 for Each of the PDE Isoform Structure

\begin{tabular}{cccc}
\hline \hline Structure & $\Delta G_{\mathrm{b}}(\mathrm{kcal} / \mathrm{mol})$ & $K_{\mathrm{i}}(\mu \mathrm{M})$ & $E_{\mathrm{d}}(\mathrm{kcal} / \mathrm{mol})$ \\
\hline PDE3 & -8.06 & 1.23 & -8.66 \\
PDE4 & -6.74 & 11.5 & -7.33 \\
PDE5 & -7.79 & 2.36 & -8.39
\end{tabular}

$\Delta G_{\mathrm{b}}$ : estimated free energy of binding, $K_{\mathrm{i}}$ : estimated inhibition constant, $E_{\mathrm{d}}$ : final docking energy. docking results $\left(\Delta G_{\mathrm{b}}\right.$ : estimated free energy of bonding, $E_{\mathrm{d}}$ : final docked energy and $K_{\mathrm{i}}$ : estimated inhibition constant) are presented in Table 1.

Among the three PDE isoforms tested, LQFM 021 inhibited PDE-3 more efficiently, as evidenced by the lowest estimated free energy of binding $\left(\Delta G_{\mathrm{b}}\right)$, the estimated inhibition constant $\left(K_{\mathrm{i}}\right)$ and the final docking energy $\left(E_{\mathrm{d}}\right)$. The calculated $K_{\mathrm{i}}$ value of LQFM 021 for PDE-3 was approximately nine- and twofold lower for PDE-4 and PDE-5, respectively (Table 1). The results in Table 1 indicate that LQFM 021 could be a possible inhibitor of PDE-3.

The major energetically favored binding clusters of LQFM 021 in the active sites of PDE-3, PDE-4 and PDE-5 are shown

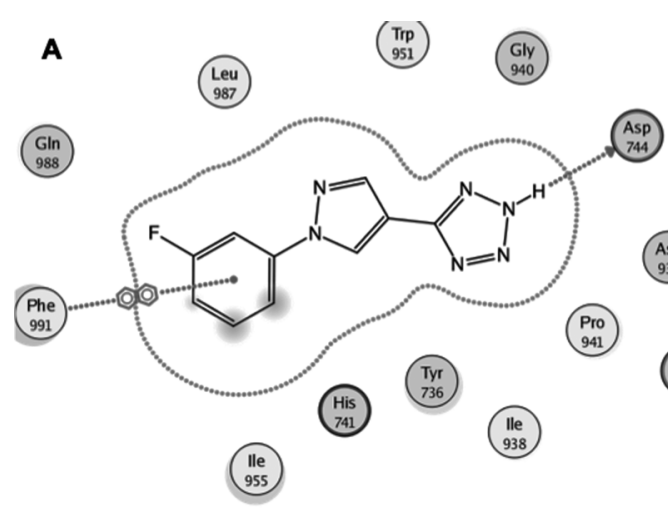

c

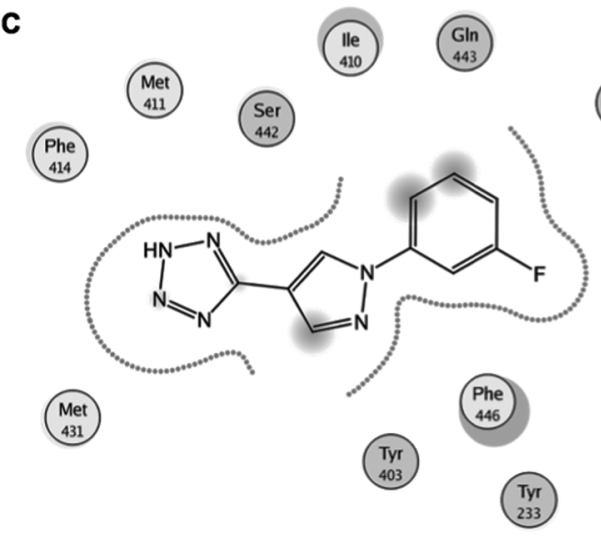

E

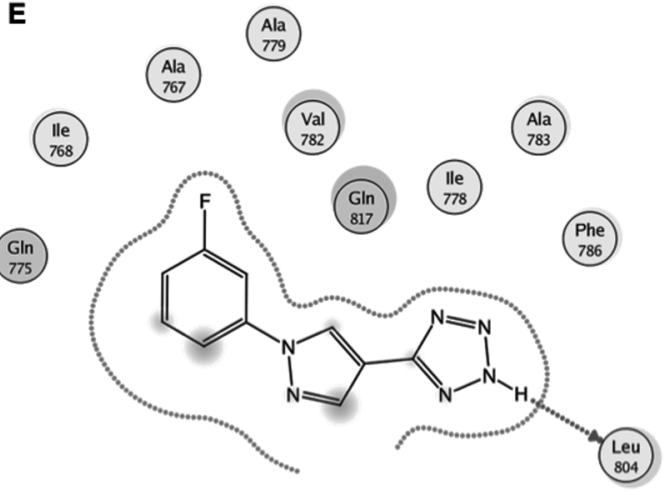

Asn
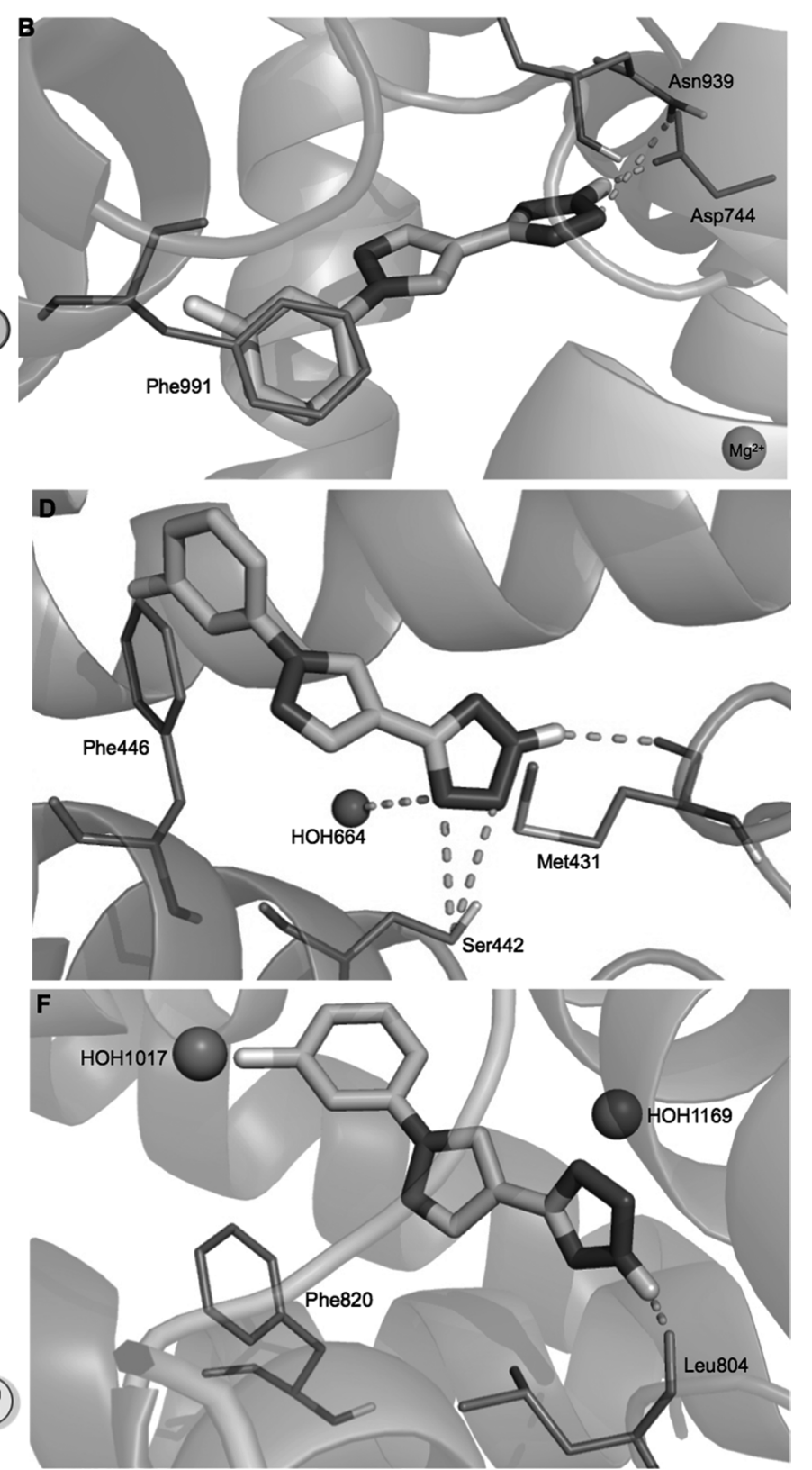

Fig. 2. The Major Energetically Favored Binding Clusters of LQFM 021 in the Active Sites of PDE-3 (A and B), PDE-4 (C and D) and PDE-5 (E and F) from Docking Studies

A, C and E show a schematic 2D diagram of active site residues and LQFM 021 interactions. B, D and F show the 3D representation of PDE structures with select amino acid residues shown as lines (the carbon atoms are in gray). The LQFM 021 is shown as sticks (the carbon atoms are shown in green). Water molecules and the magnesium ion are shown as spheres. The green dotted lines denote hydrogen bonds. (Color images were converted into gray scale.) 


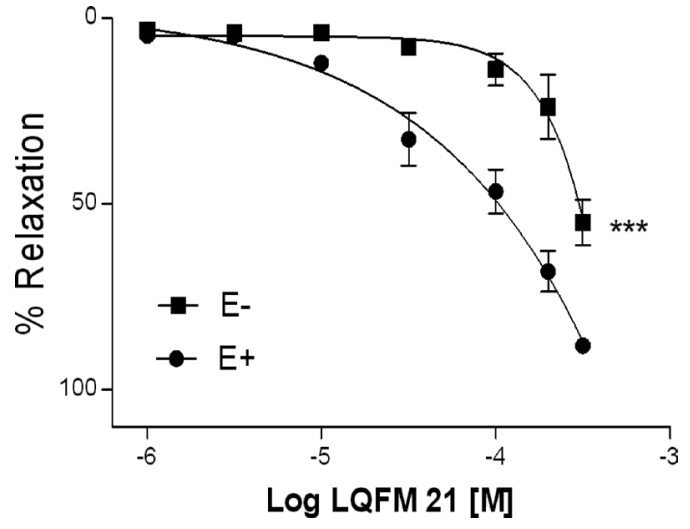

Fig. 3. Relaxant Effect of LQFM 021 in Isolated Rat Aortas

Cumulative concentration-effect curve for LQFM 021 in aortas with endothelium $\left(\mathrm{E}^{+}, n=5\right)$ and without endothelium $\left(\mathrm{E}^{-}, n=7\right)$ pre-contracted with phenylephrine $\left(\mathrm{EC}_{50}\right)$. The points represent means \pm S.E.M. of the relaxing effect expressed in percentage. Significant difference $* * * p<0.001$.

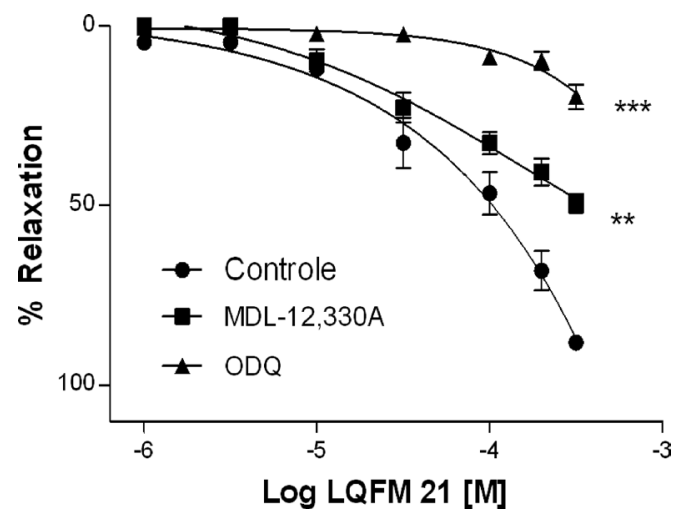

Fig. 4. Effect of MDL-12.330A and ODQ on Relaxation Induced by LQFM 021 on Isolated Aortas

Cumulative concentration-effect curves for LQFM 021 before and after ( $30 \mathrm{~min}$ ) incubation with MDL-12.330A $(10 \mu \mathrm{M})$ or ODQ $(1 \mu \mathrm{M})$ in arteries pre-contracted with phenylephrine $\left(\mathrm{EC}_{50}\right)$. The points represent means \pm S.E.M. of the relaxing effect expressed in percentage. Significant difference $* * p<0.01 ; * * * p<0.001$.

in Fig. 2. Analyzing the docked structure of LQFM 021 with PDE-3 (Figs. 2A, B), the nitrogen atoms in the tetrazole ring were noted to likely form hydrogen bonds with the Asp744 and Asn939 residues, and there is a $\pi$-stacking interaction between Phe991 and the phenyl ring, which was located in the Q-pocket. Moreover, a hydrogen bond between the fluorine atom of LQFM 021 and the NH of Gln988 was observed.

The docked orientation of LQFM 021 in PDE-4 (Figs. $2 \mathrm{C}$, D) suggested that the nitrogen atoms of the tetrazole ring might be able to form hydrogen bonds with Met431 and Ser442 and with a water molecule (664), and there is a $\pi$-stacking interaction between Phe446 and the phenyl ring. The best conformation for LQFM 021 docked in PDE-5 (Figs. $2 \mathrm{E}, \mathrm{F}$ ) was in a position to form only one hydrogen bond with Leu804.

Effects of LQFM 021 on Isolated Arteries. Relaxant Effect of LQFM 021 in the Rat Vascular Smooth Muscle According to Fig. 3, LQFM 021 added cumulatively to the bath solution during sustained contraction induced by phenylephrine evoked concentration-dependent relaxation. The value of the maximum effect to the aortas with endothelium $\left(\mathrm{E}^{+}\right)$

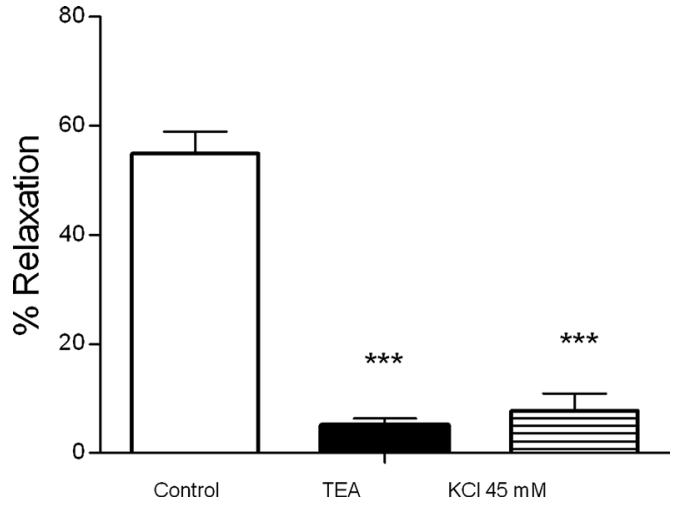

Fig. 5. Maximum Relaxation Effect for LQFM 021 in Aortas Precontracted with Phenylephrine $\left(\mathrm{EC}_{50}\right)$ before or after $(30 \mathrm{~min})$ Incubation with TEA (5 mM) or under Pre-contraction with $45 \mathrm{~mm} \mathrm{KCl}$

Vertical bars represent the mean \pm S.E.M. values of the maximum effect. Significant difference $* * * p<0.001$

was $88.1 \pm 2.1 \%(n=5)$. For aortas without endothelium $\left(\mathrm{E}^{-}\right)$, the value of the maximum effect was approximately $37.6 \%$ lower $(p<0.001)$, reaching a maximum relaxation of $54.9 \pm$ $6.0 \%(n=7)$.

Effect of Selective Inhibitors of Soluble Guanylyl Cyclase (sGC) and Adenylyl Cyclase (AC) on the Relaxation Induced by LQFM 021 The AC inhibitor MDL-12.330A and the SGC inhibitor ODQ significantly reduced the relaxing effect of LQFM 021 from $88.1 \pm 2.1 \%$ to $48.3 \pm 3.0 \%(n=5)$ and $19.9 \pm 2.3 \% \quad(n=6)$, respectively (Fig. 4). The reduction was $45.1 \%$ and $77.4 \%$ (for MDL-12,330A and ODQ, respectively) in relation to the control group (absence of inhibitor).

Effect of Membrane Depolarization with $\mathrm{KCl}$ or Blockade of the $\mathrm{K}^{+}$Channels on Relaxation Induced by LQFM 021 Figure 5 shows the maximum relaxation induced by LQFM 021 in preparations pre-contracted with phenylephrine in the absence or presence of the nonselective $\mathrm{K}^{+}$channels blocker TEA $(5 \mathrm{~mm})$ or in preparations pre-contracted with $\mathrm{KCl}(45 \mathrm{~mm})$. The treatment with the TEA altered the maximal relaxation for LQFM 021 (from $54.9 \pm 6.0 \%$ to $5.0 \pm 1.2 \%$, $n=6)$. Likewise, the pre-contraction of the preparations with $\mathrm{KCl} 45 \mathrm{~mm}$ also reduced the maximal relaxation induced by LQFM 021 to $7.8 \pm 3.1 \%(n=5)$. The difference was statistically significant $(p<0.001)$ for both treatments.

Effects of the SERCA Inhibitor CPA on the Relaxation Induced by LQFM 021 Our data showed that CPA $(10 \mu \mathrm{M})$ altered the relaxation induced by LQFM 021 (Fig. 6). The maximum effect of LQFM 021 in preparations contracted with phenylephrine $(54.9 \pm 6.0 \% \quad n=7)$ was significantly reduced $(p<0.001)$ by pre-treatment $(30 \mathrm{~min})$ with $\mathrm{CPA}$ to $19.4 \pm 4.5 \%$, $n=6$. The results with CPA suggested that calcium stored in the sarcoplasmic reticulum does have significant importance for the relaxing effect observed for LQFM 021 in arterial smooth muscle.

Effect of $\mathrm{LQFM} 021$ on $\mathrm{CaCl}_{2}$-Induced Contractions Aortic rings were used to investigate contractile responses dependent on extracellular $\mathrm{Ca}^{2+}$ influx (Fig. 7). Cumulative additions of $\mathrm{CaCl}_{2}$ produced concentration-dependent arterial smooth muscle contractions in the presence of phenylephrine. Contraction responses are expressed as a percentage of the response to contraction induced by $\mathrm{KCl}(45 \mathrm{~mm})$. The group 


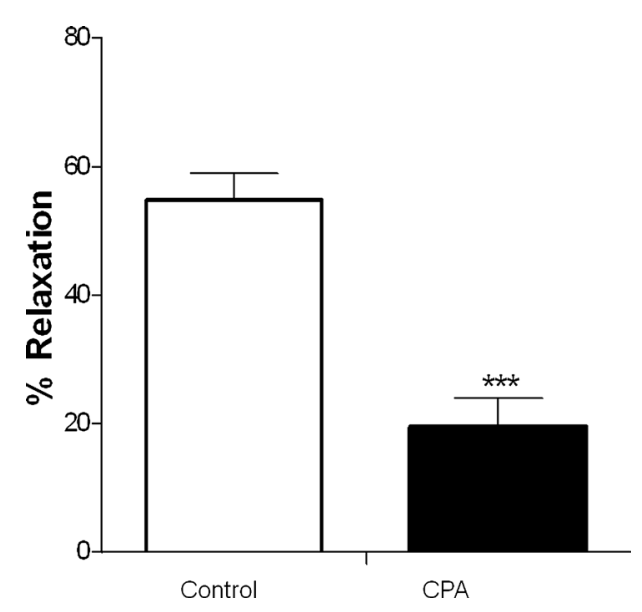

Fig. 6. Maximum Relaxation Effect for LQFM 021 in Aortas Pre-contracted with Phenylephrine $\left(\mathrm{EC}_{50}\right)$ in the Absence or Presence $(30 \mathrm{~min})$ of the $\mathrm{Ca}^{2+}$-ATPase Inhibitor CPA $(10 \mu \mathrm{M})$

Vertical bars represent the mean \pm S.E.M. values of the maximum effect. Significant difference $* * * p<0.001$.

incubated with LQFM $021(100 \mu \mathrm{M}$ for $20 \mathrm{~min})$ presented significantly decreased $(p<0.001)$ contraction (approximately $38.5 \%$ ) compared to the control group. The maximal effect of the control group was $123.1 \pm 8.0 \%(n=6)$, which was reduced to $75.5 \pm 11.2 \%(n=7)$ after treatment with LQFM 021 . These results suggested that inhibition of $\mathrm{Ca}^{2+}$ influx is an important mechanism for LQFM 021-induced arterial smooth muscle relaxation.

Evaluation of Cytotoxicity. Incorporation of Neutral Red in Basal Line 3T3 Exposure of 3T3 cells to various concentrations of LQFM $021(0.005-0.602 \mathrm{mg} / \mathrm{mL})$ resulted in a decline in cell proliferation in a concentration-dependent manner $\left(\mathrm{IC}_{50}\right.$ of $59.8 \mu \mathrm{g} / \mathrm{mL}$ ). From this $\mathrm{IC}_{50}$ value, we estimated the likely in vivo $\mathrm{LD}_{50}(533 \mathrm{mg} / \mathrm{kg})$, which helped us in choosing the initial dose of the compound to be administered for evaluation of acute oral toxicity.

Acute Oral Toxicity Evaluation of LQFM 021 Some signs of toxicity in animals after the administration of $2000 \mathrm{mg} / \mathrm{kg}$ of LQFM 021 were observed in the present study. In the first four hours, they presented apathy and subsequent paresis in the hind legs. One showed a higher motor impairment and died soon after. The others recovered completely after $24 \mathrm{~h}$. After $14 \mathrm{~d}$, no other signs of abnormality were presented until the animals were euthanized. Therefore, LQFM 021 was categorized as "Class 4" using the Global Harmonized System (GHS) (i.e., acute lethal toxicity between 300 and $2000 \mathrm{mg} / \mathrm{kg}$ ). There were no significant differences in body weight (data not shown).

\section{Discussion}

The present study demonstrated the structural design of the new heterocyclic drug LQFM 021, a possible inhibitor of PDEs (mainly PDE-3, shown here with molecular docking) and a vasodilation inducer. Part of this vasodilatatory effect was pharmacologically characterized in this work. Our results showed that this compound induced relaxation in vascular smooth muscle in the presence or absence of endothelium, and requires participation of cyclic nucleotides, internal capitation of $\mathrm{Ca}^{2+}$ by the sarcoplasmic reticulum, and $\mathrm{Ca}^{2+}$ and $\mathrm{K}^{+}$ flux across the sarcolemma. Furthermore, toxicity analysis

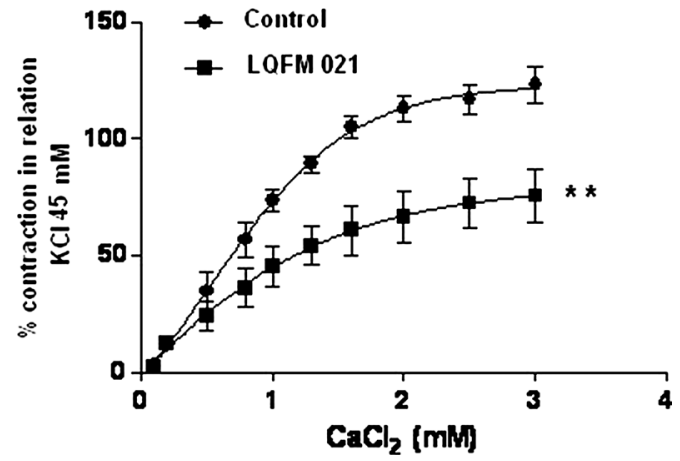

Fig. 7. Concentration-Response Curves for Extracellular Calcium $\left(\mathrm{CaCl}_{2}\right)$ in the Absence (Control, $\left.n=5\right)$ or Presence $(20 \mathrm{~min})$ of $100 \mu \mathrm{M}$ LQFM $021(n=7)$ in Rat Aortas Stimulated by Phenylephrine $\left(\mathrm{EC}_{50}\right)$

Each point represents the mean \pm S.E.M. and is expressed as maximal change from the contraction produced by $\mathrm{KCl}(45 \mathrm{~mm})$, which was taken as $100 \%$. Significant difference $* * p<0.01$.

revealed that LQFM 021 was well tolerated in oral administration.

A detailed assessment of each conformer of LQFM 021 docked in PDE-3 (all of the 150 docked conformations) revealed that more than $50 \%$ of docking results for PDE-3 had nearly identical orientations in which the following were observed: a) the fluorophenyl group was oriented toward Glu988, allowing a $\pi-\pi$ interaction with Phe991, b) the phenyl and pyrazole moieties were flanked by the hydrophobic portions of Ile938, Ile955, Leu987, Phe991 and Ile992 and c) the tetrazole moiety was surrounded by Asn939 and Asp744. Due to the orientation mentioned, the docked conformers displayed a hydrogen bond between the amide of Gln988 in PDE-3 and the fluorine atom of LQFM 021 at a distance of $2.33 \AA$ (Fig. 2). The importance of these amino acids in the catalytic activity of the PDE-3 has been proved previously by site directed mutagenesis and X-ray studies. ${ }^{18,31)}$

The endothelium can modulate vascular smooth muscle response to different contractile or relaxant stimuli. ${ }^{32)}$ In the present study, we evaluated the relaxation stimulated by LQFM 021 in arteries with and without functional endothelium in an attempt to verify the endothelial modulation in this response. The results showed that the endothelium, while not essential for relaxation, potentiated vascular relaxation stimulated by the compound. Our data corroborates other studies where the vascular endothelium also enhanced the relaxation caused by PDE inhibitors, including inhibitors of PDE-3. ${ }^{8-10}$ )

PDE-3 has a catalytic site with high affinity for cAMP and cGMP. However, the hydrolysis of cAMP is 10 times faster than that for the hydrolysis of cGMP. Therefore, cGMP effectively acts as a competitive inhibitor for cAMP and thus for PDE-3. ${ }^{33,34)}$ In our experiments, we observed that the inhibition of the AC/cAMP and sGC/cGMP pathways reduced the relaxation induced by LQFM 021 . The results also showed that the cGMP pathway seems to be more important for PDE-3 selective inhibitors, confirming the results observed by van der Zypp et al. ${ }^{10)}$ However, as observed in our results as well as other scientific findings, the inhibition of the AC/cAMP and GCs/cGMP pathways does not completely prevent relaxation stimulated by PDE inhibitors. This indicated that the vasorelaxant response stimulated by PDE-3 is at least partly independent of cyclic nucleotides. 
The opening of $\mathrm{K}^{+}$channels in vascular smooth muscle cell membranes increases the efflux of $\mathrm{K}^{+}$, resulting in hyperpolarization of the membrane. This leads to vasodilation and reduction in the cytosolic $\mathrm{Ca}^{2+}{ }^{35)}$ In the present study, LQFM 021 produced a concentration-dependent relaxation in arteries previously contracted with the $\alpha 1$-adrenergic agonist phenylephrine. However, this relaxant effect was not effective when the contractile agent was $\mathrm{KCl}$. In addition, the relaxation was virtually abolished upon preliminary treatment of the arteries with TEA, showing that the efflux of $\mathrm{K}^{+}$is important in the relaxing effect of the compound. These data are in agreement with other studies in pulmonary arteries of rabbits, ${ }^{36)}$ aortas of rats $^{37)}$ and canine mesenteric arteries and veins ${ }^{38)}$ using selective inhibitors of PDEs, including PDE-3. This suggested that the vasodilatory action of the LQFM 021 involves activation of $\mathrm{K}^{+}$channels, as observed for other selective inhibitors of PDE-3, such as milrinone and amrinone. The activation of $\mathrm{K}^{+}$ channels, leading to membrane hyperpolarization and inhibition of $\mathrm{Ca}^{2+}$ influx, is an important target of action of several vasodilator drugs. ${ }^{39)}$

Contraction and dilation of blood vessels in response to organizational demands are controlled by changes in cytosolic $\mathrm{Ca}^{2+}$ levels in vascular smooth muscle cells. Particularly for muscle contractions, the sources of $\mathrm{Ca}^{2+}$ can be intracellular, extracellular, or both. Thus, $\mathrm{Ca}^{2+}$ ions are found in four different units: extracellular, cytoplasmic, mitochondrial and non-mitochondrial (sarcoplasmic reticulum). The sarcoplasmic reticulum is the main stock of intracellular $\left.\mathrm{Ca}^{2+} .{ }^{40}\right)$ The experiments with the SERCA inhibitor CPA clearly demonstrated that the uptake of $\mathrm{Ca}^{2+}$ by the sarcoplasmic reticulum is an important step for relaxation stimulated by LQFM 021 because this relaxation decreased in the presence of CPA. The role of reticular $\mathrm{Ca}^{2+}$-ATPase pumps is to move $\mathrm{Ca}^{2+}$ to its intracellular stores and thus return plasma calcium to baseline levels after a contraction. ${ }^{41)}$

The tonic contraction induced by phenylephrine $(\alpha 1-$ adrenergic agonist) is mediated by an increased influx of extracellular $\mathrm{Ca}^{2+}$ through $\mathrm{Ca}^{2+}$ channels operated by the membrane receptor ${ }^{42}$ and voltage-sensitive channels. ${ }^{43}$ The present findings have shown that the compound LQFM 021 inhibits the contraction induced by adrenergic agonists and stimulated by external $\mathrm{Ca}^{2+}$, suggesting that LQFM 021 inhibits the influx of $\mathrm{Ca}^{2+}$ across the plasmatic membrane. These results indicated that, directly or indirectly, LQFM 021 acts as a $\mathrm{Ca}^{2+}$ channel blocker. This effect has been described for the selective inhibitors of PDE-3 amrinone and milrinone, which inhibited $\mathrm{Ca}^{2+}$ influx in aortas isolated from rabbits. ${ }^{44,45)}$

This is the first study regarding the toxicity of LQFM 021. Our results showed that LQFM 021 displayed signs of toxicity when administered in high doses $(2000 \mathrm{mg} / \mathrm{kg})$. In animals, clinical signs of apathy and paresis in hind legs were observed. The data also indicated that LQFM 021 was well tolerated when administered orally.

\section{Conclusion}

The new synthetic pyrazole LQFM 021 is a possible inhibitor of PDEs, specifically PDE-3, as shown by computational molecular docking analysis. The compound induced the relaxation of isolated arteries, an effect potentiated by the endothelium. The involvement of the sGC/cGMP and AC/cAMP pathways in this vasorelaxant effect was evident. Moreover, the flow of $\mathrm{K}^{+}$and $\mathrm{Ca}^{2+}$ through the cell membrane and the uptake of $\mathrm{Ca}^{2+}$ by the sarcoplasmic reticulum were also important for the relaxation induced by LQFM 021. LQFM 021 was well tolerated when administered orally. More experiments should be performed to elucidate the mechanism of action on the cardiovascular system and the therapeutic efficacy of LQFM 021.

Acknowledgments This study was supported by Conselho Nacional de Desenvolvimento Científico e Tecnológico (CNPq) and Coordenação de Aperfeiçoamento de Pessoal de Nível Superior (CAPES). We would like to thank Dr. Carlos Bloch Jr. of Embrapa Recursos Genéticos e Biotecnologia.

\section{References}

1) Borne R. F., "Nonsteroidal anti-inflammatory drugs," Williams and Wilkins, Baltimore, 1995.

2) Gürsoy A., Demirayak S., Capan G., Erol K., Vural K., Eur. J. Med. Chem., 35, 359-364 (2000).

3) Lincoln T. M., Cornwell T. L., Taylor A. E., Am. J. Physiol., 258, C399-C407 (1990).

4) Beavo J. A., Reifsnyder D. H., Trends Pharmacol. Sci., 11, 150-155 (1990).

5) Vandeput F., Krall J., Ockaili R., Salloum F. N., Florio V., Corbin J. D., Francis S. H., Kukreja R. C., Movsesian M. A., J. Pharmacol. Exp. Ther., 330, 884-891 (2009).

6) Boswell-Smith V., Spina D., Page C. P., Br. J. Pharmacol., 147 (Suppl. 1), S252-S257 (2006).

7) Smolenski A., J. Thromb. Haemost., 10, 167-176 (2012).

8) Mori K., Takeuchi S., Moritoki H., Tsuchiya K., Nakaya Y., Matsuoka S., Kuroda Y., Eur. Heart J., 17, 308-316 (1996).

9) Ahmad F., Degerman E., Manganiello V. C., Horm. Metab. Res., 44, 776-785 (2012).

10) van der Zypp A., Rechtman M., Majewski H., Gen. Pharmacol., 34, 245-253 (2000).

11) Minami K., Fukuzawa K., Nakaya Y., Zeng X. R., Inoue I., Life Sci., 53, 1129-1135 (1993).

12) Komalavilas P., Lincoln T. M., J. Biol. Chem., 271, 21933-21938 (1996).

13) Takuwa Y., Jpn. Heart J., 37, 793-813 (1996).

14) Wang Y. X., Poon K. S., Randall D. J., Pang C. C., Br. J. Pharmacol., 250, 335-340 (1993).

15) Cardillo C., Kilcoyne C. M., Quyyumi A. A., Cannon R. O. 3rd, Panza J. A., Hypertension, 30, 918-921 (1997).

16) OpenEye Scientific Software, Inc., "OMEGA version 2.4.6." Santa Fe, NM, U.S.A.: 〈http://www.eyesopen.com〉 2012.

17) Hawkins P. C. D., Skillman A. G., Warren G. L., Ellingson B. A., Stahl M. T., J. Chem. Inf. Model., 50, 72-584 (2010).

18) Scapin G., Patel S. B., Chung C., Varnerin J. P., Edmondson S. D., Mastracchio A., Parmee E. R., Singh S. B., Becker J. W., Van der Ploeg L. H., Tota M. R., Biochemistry, 43, 6091-6100 (2004).

19) Xu R. X., Rocque W. J., Lambert M. H., Vanderwall D. E., Luther M. A., Nolte R. T., J. Mol. Biol., 337, 355-365 (2004).

20) Card G. L., England B. P., Suzuki Y., Fong D., Powell B., Lee B., Luu C., Tabrizizad M., Gillette S., Ibrahim P. N., Artis D. R., Bollag G., Milburn M. V., Kim S. H., Schlessinger J., Zhang K. Y., Structure, 12, 2233-2247 (2004).

21) Morris G. M., Huey R., Lindstrom W., Sanner M. F., Belew R. K., Goodsell D. S., Olson A. J., J. Comput. Chem., 16, 2785-2791 (2009).

22) Fotakis G., Timbrell J. A., Toxicol. Lett., 160, 171-177 (2006).

23) Malone M. H., Robichaud R. C., Lloydia, 25, 320-332 (1962).

24) Malone M. H., "Pharmacological Approaches to Natural Products Screening and Evaluation," ed. by Wagner H., Wolf P., SpringerVerlag, Berlin, 1977, pp. 23-53.

25) Finar I. L., Godfrey K. E., J. Chem. Soc., 1954, 2293-2298 (1954) 
26) Johansson A., Abrahamsson M., Magnuson A., Huang P., Mårtensson J., Styring S., Hammarström L., Sun L., Akermark B., Inorg. Chem., 42, 7502-7511 (2003).

27) Lalehzari A., Desper J., Levy C. J., Inorg. Chem., 47, 1120-1126 (2008).

28) Ballini R., Fiorini D., Palmieri A. L., Synlett, 12, 1841-1843 (2003).

29) Zwaagstra M. E., Timmerman H., Tamura M., Tohma T., Wada Y., Onogi K., Zhang M. Q., J. Med. Chem., 40, 1075-1089 (1997).

30) Wang R., Lai L., Wang S., J. Comput. Aided Mol. Des., 16, 11-26 (2002).

31) Zhang W., Ke H., Colman R. W., Mol. Pharmacol., 62, 514-520 (2002).

32) Hyslop S., de Nucci G., Wien. Klin. Wochenschr., 103, 422-434 (1991).

33) Lugnier C., Pharmacol. Ther., 109, 366-398 (2006).

34) Shaur Y., Holst L. S., Landstrom T. R., Prog. Nucleic Acid Res. Mol. Biol., 66, 241-277 (2001).

35) Nelson M. T., Quayle J. M., Am. J. Physiol., 268, C799-C822
(1995).

36) Lee T. S., Hou X., Chest, 108, 1364-1367 (1995).

37) Lindgren S., Andersson K. E., Gen. Pharmacol., 22, 617-624 (1991).

38) Itoh H., Sato Y., Taniguchi K., Nakagawa H., Inui J., Takeuchi J., Eur. J. Pharmacol., 218, 347-349 (1992).

39) Miyoshi H., Nakaya Y., Moritoki H., FEBS Lett., 345, 47-49 (1994).

40) Thomas A. P., Bird G. St. J., Hajnóczky G., Robb-Gaspers L. D., Putney J. W. Jr., FASEB J., 10, 1505-1517 (1996).

41) Carafoli E., Annu. Rev. Biochem., 56, 395-433 (1987).

42) Hirata S., Enoki T., Kitamura R., Vinh V. H., Nakamura K., Mori K., Br. J. Anaesth., 81, 578-583 (1998).

43) Lee C. H., Poburko D., Sahota P., Sandhu J., Ruehlmann D. O., van Breemen C., J. Physiol., 534, 641-650 (2001).

44) Meisheri K. D., Palmer R. F., Van Breemen C., Eur. J. Pharmacol., 61, 159-165 (1980).

45) Tejerina T., Sesin J., Delgado C., Tamargo J., Eur. J. Pharmacol., 148, 239-246 (1998). 